\title{
A Natural Generalization of Linear Isotropic Relations with Seth-Hill Strain Tensors to Transversely Isotropic Materials at Finite Strains
}

\author{
Zhi-Qiao Wang ${ }^{1,2}$ and Yu Wang ${ }^{1,2}$ \\ ${ }^{1}$ School of Engineering and Technology, China University of Geosciences, Beijing 100083, China \\ ${ }^{2}$ Key laboratory on Deep Geo-Drilling Technology of the Ministry of Land and Resources, China University of Geosciences, \\ Beijing 100083, China \\ Correspondence should be addressed to Yu Wang; wangyu203@cugb.edu.cn
}

Received 4 March 2016; Revised 23 June 2016; Accepted 21 July 2016

Academic Editor: Jose Merodio

Copyright (c) 2016 Z.-Q. Wang and Y. Wang. This is an open access article distributed under the Creative Commons Attribution License, which permits unrestricted use, distribution, and reproduction in any medium, provided the original work is properly cited.

\begin{abstract}
Hooke's law was naturally generalized to finite strains by Hill in 1978, by introducing the Seth-Hill strain and its conjugate stress. This paper presents the transversely isotropic relations, which are not only a natural extension of Hill's theory from isotropic materials to transversely isotropic materials, but also the natural generalization of the transversely isotropic Hooke's law from infinitesimal strains to moderate strains. This generalization introduces a class of transversely isotropic hyperelastic models, which are adopted to investigate the uniaxial stretch and the simple shear problems. Results show that the material responses for different constitutive equations are significantly different; the stiffening or softening behaviors of materials at moderate deformations can be described by the appropriate model with proper material parameters.
\end{abstract}

\section{Introduction}

In the context of isotropic finite elasticity, Hooke's law for infinitesimal deformations is usually generalized to moderate deformations by adopting different stress and strain measures [1-5]. Some of the generalized linear constitutive models are hyperelastic, but some are not. Hill [4] defined a class of Hookean type hyperelastic materials by replacing the Cauchy stress and the infinitesimal strain in Hooke's law with any given Seth-Hill strain and its conjugate stress. Miehe and Lambrecht [6] developed representation of stresses and elasticity tensors for Seth-Hill strain tensor. Xiao and Chen $[7,8]$ studied a special case of Hill's materials, namely, the linear elastic Hencky model at finite deformations. Farahani and Bahai [9] analyzed the linear isotropic Hill theory and compared the results with the hypoelastic model of Cauchy stress. Xiao and $\mathrm{He}[10]$ gave a unified exact analysis for the Poynting effects of cylindrical tubes at finite strains, made of Hill's class of elastic materials. Some general results for linear isotropic relations in finite hyperelasticity are suggested by Murphy [11]. Further, Gilchrist and his coworkers [12] investigated the generalization of the linear isotropic elasticity to model biological soft tissue undergoing moderate strains. They experimentally proved that when a proper strain tensor is chosen, such generalizations of the structures of the linear theory to the nonlinear regime might provide an efficient method to model the mechanical behaviors of such materials at moderate deformations.

The researches mentioned above are mainly concerned with the isotropic cases. In many engineering problems, it is necessary to characterize the mechanical behaviors of anisotropic materials undergoing large deformations. In the past decades, there have been several different studies on the large strain responses of anisotropic materials following the pioneer work of Ericksen and Rivlin [13], which can be referred to Spencer [14], Silhavy [15], Holzapfel [16], and references therein. To the authors' knowledge, there are mainly three methods to construct the anisotropic hyperelastic constitutive relations at finite strains. The first approach is the introduction of some specific nonlinear elastic strain energy 
functions, which are functions of the irreducible invariants of strain tensor and structure tensor [13-20]; in particular, Merodio and the coworkers [21, 22] use this approach to deal with different invariants, anisotropic materials, residual stress, and structural instabilities. The second is based on the fictitious configuration; Menzel and Steinmann [23] assumed that there is a fictitious isotropic configuration that renders an anisotropic undeformed reference configuration via an appropriate linear tangent map. Then, the constitutive equations are built up based on the strain energy equivalence. The third is based on the projection method; Mahnken [24] developed the anisotropic geometrically nonlinear elastic model with generalized Seth-Hill strain tensors projected to invariant subspaces. The Seth-Hill strain tensors are widely used not only in anisotropic finite elasticity, but also in anisotropic finite elastoplasticity [25-27]. The present paper focuses on the cases of finite elasticity and adopts the first approach, similar to isotropic cases [1-13], to generalizing the structures of the classical anisotropic linear theory to moderate deformation cases. The intuitive expectation for doing so is that, given the excellent agreement of the classical model with experimental data, such generalizations can provide simple and accurate models of nonlinear elastic media undergoing moderate deformations [12]. Therefore, the linear anisotropic elasticity with Seth-Hill strain tensors for moderate strains is worthy of further study.

The present paper is not only a natural extension of Hill's relations from isotropic materials to transversely isotropic materials, but also the natural generalization of transversely isotropic Hooke's law from infinitesimal strains to moderate strains. The outline of the paper is as follows. Section 2 briefly recalls the Seth-Hill strain tensors and their conjugate stress tensors. Section 3 naturally generalizes Hill's class of Hookean compressible materials to transversely isotropic materials. Finally, the uniaxial stretch and the simple shear are analyzed by using these generalized models; the stiffening or softening behaviors of such materials are discussed. The results may be useful for the mechanical analysis of soft tissue materials.

\section{Seth-Hill Strain Tensors and Their Conjugate Stress Tensors}

2.1. Strain Measures. For a deformation body, with $\mathbf{F}$ denoting the deformation gradient at a material point, the polar decomposition theorem of $\mathbf{F}$ states can be

$$
\mathbf{F}=\mathbf{R} \cdot \mathbf{U}=\mathbf{V} \cdot \mathbf{R}
$$

where $\mathbf{U}$ and $\mathbf{V}$ are the right and left stretch tensors, respectively, and $\mathbf{R}$ is the rotation tensor.

The Seth-Hill Lagrangian strain tensors are defined as

$$
\mathbf{E}^{(m)}=\frac{1}{m}\left(\mathbf{U}^{(m)}-\mathbf{I}\right)=\sum_{i=1}^{3} f\left(\lambda_{i}\right) \mathbf{N}_{i} \otimes \mathbf{N}_{i}
$$

in which $f\left(\lambda_{i}\right)=\left(\lambda_{i}^{m}-1\right) / m, \lambda_{i}$ and $\mathbf{N}_{i}$ are the principal stretches and corresponding orthonormal eigenvectors of $\mathbf{U}$, respectively, $\mathbf{I}$ is the identity tensor, and $m$ is a real number.
The corresponding Eulerian strain tensors are expressed as

$$
\boldsymbol{\varepsilon}^{(m)}=\frac{1}{m}\left(\mathbf{V}^{(m)}-\mathbf{I}\right)=\sum_{i=1}^{3} f\left(\lambda_{i}\right) \mathbf{n}_{i} \otimes \mathbf{n}_{i}
$$

in which $\mathbf{n}_{i}, i=1,2,3$, are the orthonormal eigenvectors of $\mathbf{V}$ corresponding to eigenvalues $\lambda_{i}$.

When the parameter $m=0$ in (2) and (3), we obtain the Lagrangian and the Eulerian logarithmic strains, respectively, as follows:

$$
\begin{aligned}
& \mathbf{E}^{(0)}=\ln \mathbf{U}, \\
& \boldsymbol{\varepsilon}^{(0)}=\ln \mathbf{V} .
\end{aligned}
$$

The relation between $\mathbf{E}^{(0)}$ and $\boldsymbol{\varepsilon}^{(0)}$ is

$$
\ln \mathbf{V}=\mathbf{R}(\ln \mathbf{U}) \mathbf{R}^{T} \text {. }
$$

2.2. Conjugate Stress Tensors. Hill [4] has stated that the stress measure $\mathbf{T}^{(m)}$ is said to be conjugated to a strain measure $\mathbf{E}^{(m)}$, when $\mathbf{T}^{(m)}$ and $\mathbf{E}^{(m)}$ satisfy

$$
\dot{W}=J \boldsymbol{\sigma}: \mathbf{D}=\mathbf{T}^{(m)}: \dot{\mathbf{E}}^{(m)},
$$

where $J, \boldsymbol{\sigma}$, and $\mathbf{D}$ represent the volume ratio between before and after deformation, the Cauchy stress, and strain rate tensor, respectively.

When the hyperelastic constitutive equations for the Seth-Hill strains and their conjugate stresses are adopted to solve the actual problems, it is necessary to express the Cauchy stress $\boldsymbol{\sigma}$ as $\mathbf{T}^{(m)}$ for analyzing the actual stress state at one material point. The relations of the Eulerian components of $\boldsymbol{\sigma}$ and the Lagrangian components of $\mathbf{T}^{(m)}$ are given in the following.

The left and right Cauchy-Green deformation tensors $\mathbf{B}$ and $\mathbf{C}$ possess the same principal stretches $\Lambda_{i}\left(\Lambda_{i}=\lambda_{i}^{2}\right)$ and the corresponding orthonormal eigenvectors $\mathbf{n}_{i}$ and $\mathbf{N}_{i}$, respectively. Then $\mathbf{B}$ and $\mathbf{C}$ can be expressed as the following spectral forms:

$$
\begin{aligned}
& \mathbf{B}=\Lambda_{i} \mathbf{n}_{i} \otimes \mathbf{n}_{i}, \\
& \mathbf{C}=\Lambda_{i} \mathbf{N}_{i} \otimes \mathbf{N}_{i} .
\end{aligned}
$$

$\mathbf{T}^{(m)}$ conjugated to the Seth-Hill strain tensor $\mathbf{E}^{(m)}$ can be written as

$$
\mathbf{T}^{(m)}=T_{i j}^{(m)} \mathbf{N}_{i} \otimes \mathbf{N}_{j} .
$$

Decomposing the Cauchy stress $\boldsymbol{\sigma}$ in the Eulerian principal axes, we obtain

$$
\boldsymbol{\sigma}=\bar{\sigma}_{i j} \mathbf{n}_{i} \otimes \mathbf{n}_{j}
$$

From (2) and (6), we get [29]

$$
\bar{\sigma}_{i j}=\frac{2 \lambda_{(i)} \lambda_{(j)} \varphi\left(\lambda_{(i)}, \lambda_{(j)}\right)}{J\left[\lambda_{(i)}+\lambda_{(j)}\right]} T_{i j}^{(m)}
$$

$\left(\lambda_{(i)}\right.$ represents no summation), 
where

$$
\varphi\left(\lambda_{i}, \lambda_{j}\right)= \begin{cases}f^{\prime}\left(\lambda_{i}\right) & \left(\lambda_{i}=\lambda_{j}\right) \\ \frac{f\left(\lambda_{j}\right)-f\left(\lambda_{i}\right)}{\lambda_{j}-\lambda_{i}} & \left(\lambda_{i} \neq \lambda_{j}\right) .\end{cases}
$$

When $m=0$, (10) becomes

$$
\bar{\sigma}_{i j}= \begin{cases}\frac{T_{i j}^{(0)}}{J} & \left(\lambda_{i}=\lambda_{j}\right) \\ \frac{2 \lambda_{(i)} \lambda_{(j)}\left(\ln \lambda_{(j)}-\ln \lambda_{(i)}\right)}{J\left[\lambda_{(j)}^{2}-\lambda_{(i)}^{2}\right]} T_{i j}^{(0)} & \left(\lambda_{i} \neq \lambda_{j}\right) .\end{cases}
$$

The Cauchy stress $\boldsymbol{\sigma}$ can be written as $\boldsymbol{\sigma}=\sigma_{i j} \mathbf{e}_{i} \otimes \mathbf{e}_{j}$ in the physical frames. Then, the physical components of the Cauchy stress are

$$
\sigma_{i j}=\bar{\sigma}_{m n} n_{m i} n_{n j}
$$

in which $n_{m i}=\mathbf{n}_{m} \cdot \mathbf{e}_{i}, n_{n j}=\mathbf{n}_{n} \cdot \mathbf{e}_{j}$.

\section{Natural Extensions of Hill's Class of Compressible Materials}

In this section, we will give a natural extension of Hill's class of Hookean compressible materials to transversely isotropic materials at finite strains by the structure tensor method.

3.1. Hill's Class of Compressible Materials. For the isotropic linear hyperelastic materials at finite deformations, we take

$$
W=\frac{1}{2} a^{\prime} I_{1}^{2}+b^{\prime} I_{2}
$$

where $I_{1}$ and $I_{2}$ are two invariants of $\mathbf{E}^{(m)}$,

$$
\begin{aligned}
& I_{1}=\operatorname{tr}\left(\mathbf{E}^{(m)}\right), \\
& I_{2}=\operatorname{tr}\left[\left(\mathbf{E}^{(m)}\right)^{2}\right],
\end{aligned}
$$

$a^{\prime}$ and $b^{\prime}$ are Lamé constants

$$
\begin{aligned}
a^{\prime} & =\frac{E v}{(1+v)(1-2 v)}, \\
b^{\prime} & =\frac{E}{2(1+v)}
\end{aligned}
$$

with the Poisson ratio $v$ and Young's modulus $E$.

From (6) and (14), Hill's class of compressible materials is given by

$$
\mathbf{T}^{(m)}=\mathbf{C}_{I}: \mathbf{E}^{(m)},
$$

where $\mathbf{C}_{I}$ is the elasticity tensor for isotropic materials

$$
\mathbf{C}_{I}=a^{\prime} \mathbf{I} \otimes \mathbf{I}+b^{\prime}(\mathbf{I} \otimes \mathbf{I}+\mathbf{I} \widehat{\otimes} \mathbf{I}) .
$$

In (18) the fourth-order tensors $\mathbf{P} \otimes \mathbf{Q}, \mathbf{P} \otimes \mathbf{Q}$, and $\mathbf{P} \widehat{\otimes} \mathbf{Q}$ are three tensor products of the second-order tensors $\mathbf{P}$ and $\mathbf{Q}$ and defined as

$$
\begin{aligned}
& (\mathbf{P} \otimes \mathbf{Q}): \mathbf{A}=\operatorname{tr}\left(\mathbf{A} \cdot \mathbf{Q}^{T}\right) \mathbf{P}, \\
& (\mathbf{P} \otimes \mathbf{Q}): \mathbf{A}=\mathbf{P} \cdot \mathbf{A} \cdot \mathbf{Q}^{T}, \\
& (\mathbf{P} \widehat{\otimes} \mathbf{Q}): \mathbf{A}=\mathbf{P} \cdot \mathbf{A}^{T} \cdot \mathbf{Q}^{T},
\end{aligned}
$$

in which $\mathbf{A}$ is a second-order tensor.

The equivalent component formulation (17) with respect to the Lagrangian principal axes is

$$
T_{i j}^{(m)}=\left[a^{\prime} I_{1}+2 b^{\prime} f\left(\lambda_{(i)}\right)\right] \delta_{i j},
$$

where

$$
\delta_{i j}= \begin{cases}1 & i=j \\ 0 & i \neq j .\end{cases}
$$

From (10) (12), the corresponding component formulation of (17) relative to the Eulerian principal axes is

$$
J \bar{\sigma}_{i j}=\lambda_{(i)} f^{\prime}\left(\lambda_{(i)}\right)\left[a^{\prime} I_{1}+2 b^{\prime} f\left(\lambda_{(i)}\right)\right] \delta_{i j} .
$$

3.2. Transversely Isotropic Hill's Compressible Materials. In this subsection we give a natural extension of Hill's class of compressible materials to transversely isotropic materials by introducing the structure tensor $\mathbf{L}$, which is defined as

$$
\mathbf{L}=\mathbf{l}_{0} \otimes \mathbf{l}_{0},
$$

where $\mathbf{l}_{0}$ is a unit vector describing the local fiber direction in the undeformed configuration. The strain energy function $W$ is an isotropic scalar function on the strain tensor $\mathbf{E}^{(m)}$ and the structure tensor L. For geometrically nonlinear transversely isotropic materials, $W$ takes the following form:

$$
W=\frac{1}{2} a I_{1}^{2}+b I_{2}+c I_{4}^{2}+d I_{1} I_{4}+e I_{5}
$$

in which $a, b, c, d$, and $e$ are material parameters and $I_{4}$ and $I_{5}$ are two invariants of $\mathbf{E}^{(m)}$ and $\mathbf{L}$ :

$$
\begin{aligned}
& I_{4}=\operatorname{tr}\left[\mathbf{E}^{(m)} \cdot \mathbf{L}\right], \\
& I_{5}=\operatorname{tr}\left[\left(\mathbf{E}^{(m)}\right)^{2} \cdot \mathbf{L}\right] .
\end{aligned}
$$

Then, we have

$$
\mathbf{T}^{(m)}=\frac{\partial W}{\partial \mathbf{E}^{(m)}}=\mathbf{C}_{T}: \mathbf{E}^{(m)},
$$

where $\mathbf{C}_{T}$ is the elasticity tensor for transversely isotropic material

$$
\begin{aligned}
\mathbf{C}_{T}= & a \mathbf{I} \otimes \mathbf{I}+b(\mathbf{I} \otimes \mathbf{I}+\mathbf{I} \widehat{\otimes} \mathbf{I})+2 c \mathbf{L} \otimes \mathbf{L} \\
& +d(\mathbf{I} \otimes \mathbf{L}+\mathbf{L} \otimes \mathbf{I}) \\
& +\frac{e}{2}(\mathbf{I} \otimes \mathbf{L}+\mathbf{I} \widehat{\otimes} \mathbf{L}+\mathbf{L} \otimes \mathbf{I}+\mathbf{L} \widehat{\otimes} \mathbf{I}) .
\end{aligned}
$$


The component form of the constitutive model (26) with respect to the Lagrangian principal axes is

$$
\begin{aligned}
T_{i j}^{(m)}= & {\left[a I_{1}+2 b f\left(\lambda_{(i)}\right)+d I_{4}\right] \delta_{i j} } \\
& +\left\{2 c I_{4}+d I_{1}+e\left[f\left(\lambda_{(i)}\right)+f\left(\lambda_{(j)}\right)\right]\right\} l_{0 i} l_{0 j},
\end{aligned}
$$

where $l_{0 i}=\mathbf{l}_{0} \cdot \mathbf{N}_{i}$.

From (10) and (28), the corresponding component formulation of (26) relative to the Eulerian principal axes can be expressed as

$$
J \bar{\sigma}_{i j}=\frac{2 \lambda_{(i)} \lambda_{(j)} \varphi\left(\lambda_{(i)}, \lambda_{(j)}\right)}{\left(\lambda_{(i)}+\lambda_{(j)}\right)} T_{i j}^{(m)} .
$$

The present model should be able to be used in small strain cases. Engineering parameters $\left(E, E^{\prime}, v, v^{\prime}\right.$, and $\left.\mu^{\prime}\right)$ are usually used in infinitesimal strain cases, which are elastic modulus, Poisson's ratios, and shear modulus in the isotropic plane and the direction of the axis of symmetry, respectively. The relations between material parameters $a, b, c, d, e$ and engineering constants $\left(E, E^{\prime}, v, v^{\prime}\right.$, and $\left.\mu^{\prime}\right)$ are

$$
\begin{aligned}
& a=\frac{E\left(v+\xi v^{\prime 2}\right)}{\varsigma(1+v)}, \\
& b=\frac{E}{2(1+v)}, \\
& c=\frac{E^{\prime}(1-v)}{2 \varsigma}-\frac{a}{2}-b-d-e, \\
& d=\frac{E v^{\prime}}{\varsigma}-a, \\
& e=\mu^{\prime}-b,
\end{aligned}
$$

where $\xi$ and $\varsigma$ are

$$
\begin{aligned}
& \xi=\frac{E}{E^{\prime}}, \\
& \varsigma=1-v-2 \xi v^{\prime 2} .
\end{aligned}
$$

When the materials are isotropic, namely, $E=E^{\prime}, v=$ $v^{\prime}, \mu=\mu^{\prime}$, the nonvanishing parameters are $a$ and $b$, which reduce to the form of (16).

3.3. Models for Logarithmic Strains. When $m=0$, the generalized Seth-Hill strain tensor reduces to the logarithmic strain. Then, we have

$$
\mathbf{T}^{(0)}=\mathbf{C}_{T}: \ln \mathbf{U}
$$

The corresponding component form of (32) relative to Lagrangian principal axes is

$$
\begin{aligned}
T_{i j}^{(0)}= & \left(a I_{1}+2 b \ln \lambda_{(i)}+d I_{4}\right) \delta_{i j} \\
& +\left[2 c I_{4}+d I_{1}+e\left(\ln \lambda_{(i)}+\ln \lambda_{(j)}\right)\right] l_{0 i} l_{0 j} .
\end{aligned}
$$

From (10) (12), we get

$$
J \bar{\sigma}_{i j}=\frac{2 \lambda_{(i)} \lambda_{(j)} \varphi\left(\lambda_{(i)}, \lambda_{(j)}\right)}{\left(\lambda_{(i)}+\lambda_{(j)}\right)} T_{i j}^{(0)} .
$$

In the isotropic case, we have $a=a^{\prime}$ and $b=b^{\prime}$. Then, (32) and (33) are simplified to

$$
\begin{aligned}
& \mathbf{T}^{(0)}=a \operatorname{tr}(\ln \mathbf{U}) \mathbf{I}+2 b \ln \mathbf{U}, \\
& T_{i j}^{(0)}=\left(a I_{1}+2 b \ln \lambda_{(i)}\right) \delta_{i j} .
\end{aligned}
$$

Substituting (36) into (34), we obtain

$$
J \bar{\sigma}_{i j}=\left(a I_{1}+2 b \ln \lambda_{(i)}\right) \delta_{i j} .
$$

Comparing (36) and (37), we get that

$$
T_{i j}^{(0)}=J \bar{\sigma}_{i j}
$$

which implies that

$$
\mathbf{T}^{(0)}=J \mathbf{R}^{T} \cdot \boldsymbol{\sigma} \cdot \mathbf{R} .
$$

From (5), (35), and (39), we have

$$
J \boldsymbol{\sigma}=\operatorname{atr}(\ln \mathbf{V}) \mathbf{I}+2 b \ln \mathbf{V} .
$$

Considering (35) and (40), we can find that for isotropic materials the Lagrangian and Eulerian Hencky models take the same linear structures.

\section{Applications and Discussions}

In this section, the proposed transversely isotropic Hill class of compressible materials is used to analyze two kinds of homogeneous deformations, the uniaxial stretch and the simple shear.

4.1. Uniaxial Stretch. Let $\left(X_{1}, X_{2}, X_{3}\right)$ and $\left(x_{1}, x_{2}, x_{3}\right)$ be the Lagrangian and Eulerian rectangular coordinates, respectively. They possess the same origin. Without losing of generality, we consider a cubic element of transversely isotropic materials with arrises aligned with coordinate directions. Under the action of the normal stress $\sigma_{11}$ along the $x_{1}$ direction, it deforms

$$
\begin{aligned}
& x_{1}=\lambda_{1} X_{1}, \\
& x_{2}=\lambda_{2} X_{2}, \\
& x_{3}=\lambda_{3} X_{3} .
\end{aligned}
$$

Then, we have

$$
\begin{aligned}
\mathbf{E}^{(m)}= & \mathbf{e}^{(m)} \\
= & \frac{\lambda_{1}^{m}-1}{m} \mathbf{e}_{1} \otimes \mathbf{e}_{1}+\frac{\lambda_{2}^{m}-1}{m} \mathbf{e}_{2} \otimes \mathbf{e}_{2}+\frac{\lambda_{3}^{m}-1}{m} \mathbf{e}_{3} \\
& \otimes \mathbf{e}_{3}, \\
\ln \mathbf{U}= & \ln \mathbf{V} \\
= & \ln \lambda_{1} \mathbf{e}_{1} \otimes \mathbf{e}_{1}+\ln \lambda_{2} \mathbf{e}_{2} \otimes \mathbf{e}_{2}+\ln \lambda_{3} \mathbf{e}_{3} \otimes \mathbf{e}_{3},
\end{aligned}
$$


where $\mathbf{e}_{i}(i=1,2,3)$ denotes the unit vector along the $x_{i}$ direction. It is easy to get

$$
\begin{aligned}
J & =\lambda_{1} \lambda_{2} \lambda_{3}, \\
I_{1} & =\frac{\lambda_{1}^{m}+\lambda_{2}^{m}+\lambda_{3}^{m}-3}{m} .
\end{aligned}
$$

4.1.1. Uniaxial Stretch in the Fiber Direction. Let $\mathbf{1}_{0}=\mathbf{e}_{1}$, and then (41) describes the uniaxial stretch in the fiber direction. Then, we obtain $I_{4}=\left(\lambda_{1}^{m}-1\right) / m$. Considering $\sigma_{22}=\sigma_{33}=0$, from (13), (28), (29), (42), and (43), we get

$$
\lambda_{2}=\lambda_{3}=\left[1+\left(1-\lambda_{1}^{m}\right) \frac{a+d}{2(a+b)}\right]^{1 / m} .
$$

The total load acting on the element is $L=\lambda_{2} \lambda_{3} \sigma_{11}$. Then, the nondimensional total load is

$$
\frac{L}{b}=\beta_{1} \frac{\lambda_{1}^{m-1}\left(\lambda_{1}^{m}-1\right)}{m},
$$

where

$$
\beta_{1}=\left[2+\frac{2}{b}(c+d+e)+\frac{a b-2 a d-d^{2}}{b(a+b)}\right] .
$$

When $m=0$, taking limits as $m \rightarrow 0$ in (44)-(46), we obtain

$$
\begin{aligned}
\lambda_{2} & =\lambda_{3}=\lambda_{1}^{-(a+d) / 2(a+b),} \\
\frac{L}{b} & =\beta_{1} \frac{\ln \lambda_{1}}{\lambda_{1}} .
\end{aligned}
$$

4.1.2. Uniaxial Stretch in the Transverse Direction. When $\mathbf{l}_{0}=$ $\mathbf{e}_{2}$ (or $\left.\mathbf{l}_{0}=\mathbf{e}_{3}\right)$, (41) describes uniaxial stretch in the transverse direction. Herein, we only consider $\mathbf{l}_{0}=\mathbf{e}_{2}$. Then, we obtain that $I_{4}=\left(\lambda_{2}^{m}-1\right) / m$. From the boundary conditions that $\sigma_{22}=\sigma_{33}=0$, we get

$$
\begin{aligned}
& \lambda_{2}=\left[1+\left(1-\lambda_{1}^{m}\right) \frac{2 b(a+d)}{\chi}\right]^{1 / m}, \\
& \lambda_{3}=\left[1+\left(1-\lambda_{1}^{m}\right) \frac{2 a(b+c+e)-d^{2}}{\chi}\right]^{1 / m},
\end{aligned}
$$

where

$$
\chi=2 a(c+e)+4 b(a+b+c+d+e)-d^{2}
$$

The nondimensional total load acting on the element is

$$
\frac{L}{b}=\beta_{2} \frac{\lambda_{1}^{m-1}\left(\lambda_{1}^{m}-1\right)}{m}
$$

where

$$
\beta_{2}=\left[2-\frac{2 d^{2}-4 a(b+c+e)}{\chi}\right] .
$$

For the special case $m=0$, we take limits as $m \rightarrow 0$ in (48)(50) and obtain

$$
\begin{aligned}
& \lambda_{2}=\lambda_{1}^{-2 b(a+d) / \chi}, \\
& \lambda_{3}=\lambda_{1}^{\left(d^{2}-2 a(b+c+e)\right) / \chi}, \\
& \frac{L}{b}=\beta_{2} \frac{\ln \lambda_{1}}{\lambda_{1}} .
\end{aligned}
$$

4.1.3. Simplification for Isotropic Materials. For isotropic materials, the structure tensor is $\mathbf{1}_{0}=0$. Then (44), (45), (48), and (50) reduce

$$
\begin{aligned}
\lambda_{2} & =\lambda_{3}=\left[1+\left(1-\lambda_{1}^{m}\right) \frac{a}{2(a+b)}\right]^{1 / m} \\
& =\left[1+\left(1-\lambda_{1}^{m}\right) v\right]^{1 / m} \\
\frac{L}{b} & =\beta_{0} \frac{\lambda_{1}^{m-1}\left(\lambda_{1}^{m}-1\right)}{m}
\end{aligned}
$$

in which

$$
\beta_{0}=2+\frac{a}{a+b} .
$$

When $m=0$, (47) and (52) reduce

$$
\begin{aligned}
\lambda_{2} & =\lambda_{3}=\lambda_{1}^{-a / 2(a+b)}=\lambda_{1}^{-v}, \\
\frac{L}{b} & =\beta_{0} \frac{\ln \lambda_{1}}{\lambda_{1}}=2(1+v) \frac{\ln \lambda_{1}}{\lambda_{1}} .
\end{aligned}
$$

4.1.4. Discussions. Results of the uniaxial stretch are given above for Hill's class of materials for several numbers $m$. Noting (45), (50), and (54), it is easy to find that they take the same structures with different material parameters $\beta_{i}, i=$ $0,1,2$. Hence, we give the unified expressions of nondimensional total force

$$
\frac{L}{b}=\beta_{i} \frac{\lambda_{1}^{m-1}\left(\lambda_{1}^{m}-1\right)}{m} .
$$

Now, we study how the nondimensional total force $L / b$ will change. Hence, we consider the derivative of $L / b$ with respect to $\lambda_{1}$,

$$
D\left[\frac{L}{b}\right]=\beta_{i} \lambda_{1}^{m-2}\left[\lambda_{1}^{m}\left(2-\frac{1}{m}\right)-\left(1-\frac{1}{m}\right)\right] .
$$

Firstly, we consider the tensile case where $\lambda_{1}>1$. When $m \geq 1 / 2$, (58) suggests that $D[L / b]>0$ which predicts that the nondimensional total force $L / b$ will increase monotonously in the process of stretching. When $-\infty<m<1 / 2$, from $D[L / b]=0$, we can obtain that the tensile force $L / b$ will reach its maximum $\beta_{i}(1 /(1-2 m))((m-1) /(2 m-1))^{1-1 / m}$ at $\lambda_{1}=((m-1) /(2 m-1))^{1 / m}$.

Secondly, we analyze the compression case in which $0<$ $\lambda_{1}<1$. When $-\infty<m \leq 1$, (58) suggests that $D[L / b]>0$ which predicts that the compressive force $L / b$ will increase 


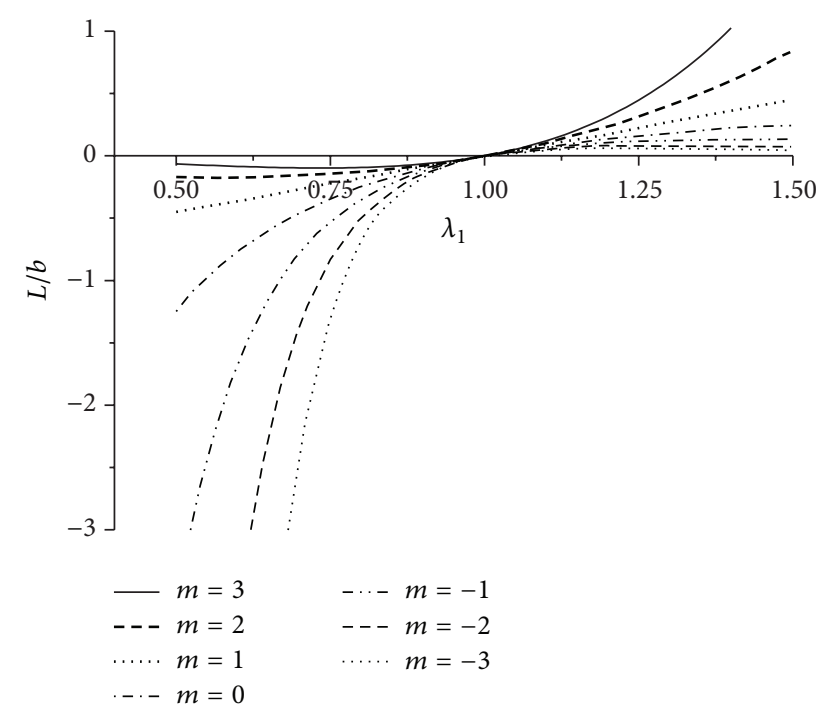

Figure 1: Relations between nondimensional loading $L / b$ and stretch $\lambda_{1}$ for $\mathbf{l}_{0}=\mathbf{e}_{1}$.

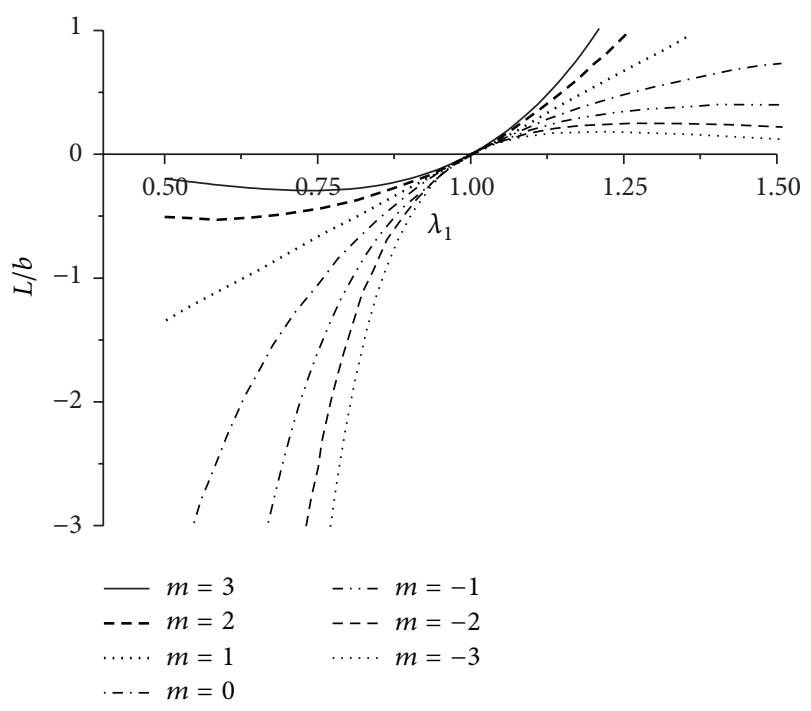

FIGURE 2: Relations between nondimensional loading $L / b$ and stretch $\lambda_{1}$ for $\mathbf{l}_{0}=\mathbf{e}_{2}$.

monotonously in the process of compressing. When $1<m<$ $\infty$, from $D[L / b]=0$, we can obtain that the compressive force $L / b$ will reach its maximum $\beta_{i}(1 /(1-2 m))((m-1) /(2 m-$ $1))^{1-1 / m}$ at $\lambda_{1}=((m-1) /(2 m-1))^{1 / m}$.

In order to analyze the differences between the proposed models with the different number $m$, we draw the relations between the nondimensional total load and uniaxial stretch for the known models with $m=0, \pm 1, \pm 2, \pm 3$ in Figures 1 and 2 in which the parameters $v, v^{\prime}, \xi$, and $\mu^{\prime} / b$ in (30) are $0.35,0.25,3$, and 0.5 , respectively. Figure 1 shows the relations between the nondimensional total load $L / b$ and the uniaxial

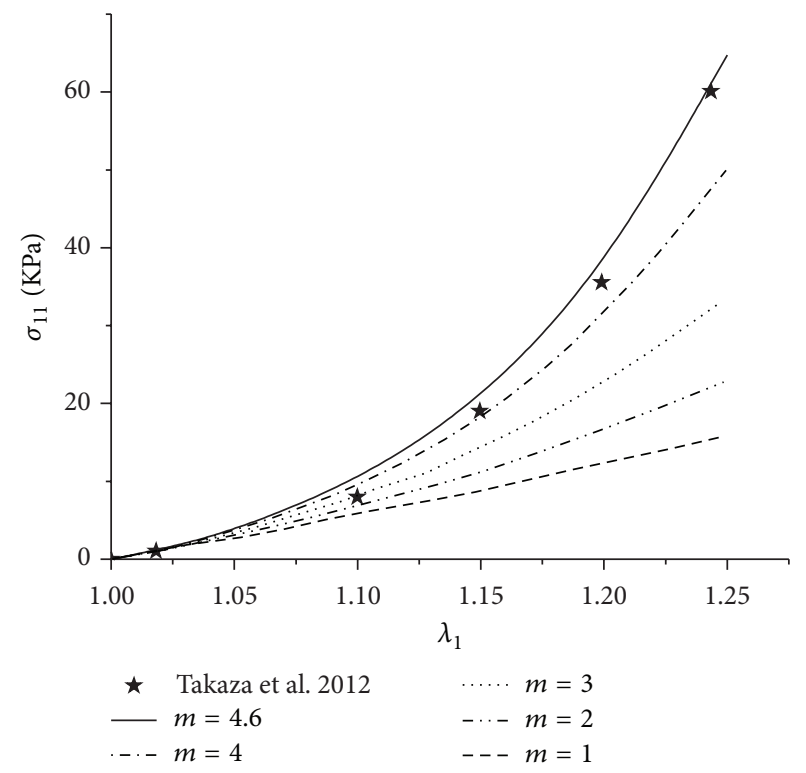

FIgURE 3: Comparison of the present results with experimental data [28] (stretch in the fiber direction).

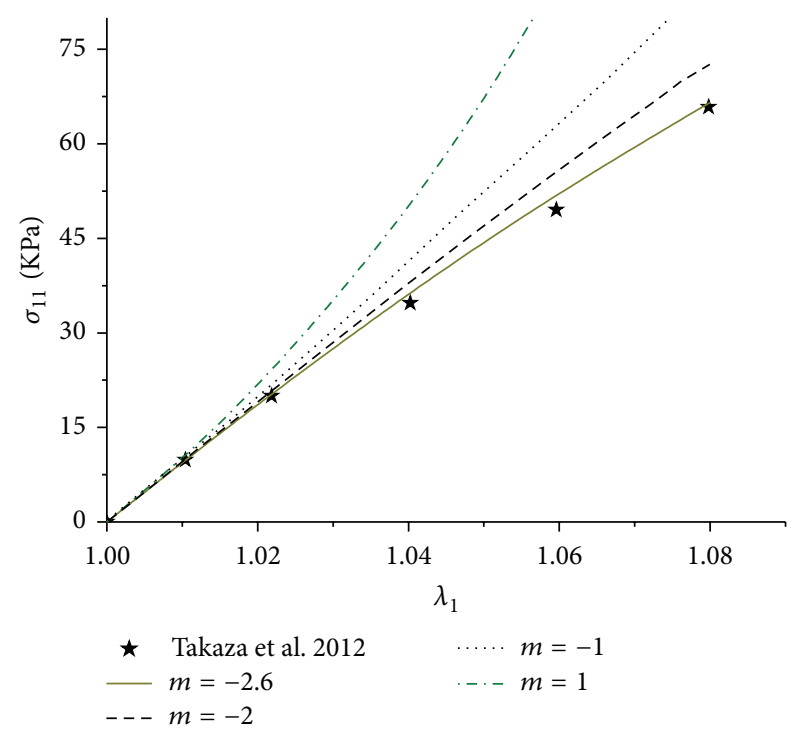

FIGURE 4: Comparison of the present results with experimental data [28] (stretch in the transverse direction).

stretch $\lambda_{1}$ when $\mathbf{l}_{0}=\mathbf{e}_{1}$. When $\mathbf{l}_{0}=\mathbf{e}_{2}$, results are given in Figure 2. It can be found that when $m>1$ the material stiffens in tension and softens in compression. When $m=1$, it predicts the linear relations. When $m<1$ the material shows tensile softening and compressive stiffening.

In applications, once engineering constants $\left(E, E^{\prime}, v, v^{\prime}\right.$, and $\left.\mu^{\prime}\right)$ are obtained from experimental data, we can give parameters $a, b, c, d$, and $e$ for moderate deformations from (30). Then, the exponent " $m$ " can be determined by fitting the experimental data. Figures 3 and 4 show the comparison 
between the present results and experimental data [28] of the uniaxial stretch in the fiber longitudinal and transverse directions, respectively. In reference [28], the values of $v$ and $v^{\prime}$ are measured. From the uniaxial stretch experimental data (in fiber longitudinal and transverse two directions), we can obtain parameters $E$ and $E^{\prime}$, respectively. At last, we determined the parameter $\mu^{\prime}$ and the exponent " $m$ " by fitting the experimental data. We get the material parameters $a=$ $31.1763 \mathrm{KPa}, b=17.1474 \mathrm{KPa}, c=450.692 \mathrm{KPa}, d=$ $-4.11503 \mathrm{KPa}$, and $e=0$. It can be found that when $m=4.6$ for stretch fiber longitudinal directions (and $m=-2.6$ for stretch in transverse directions), present results agree well with the experiment data. The tissue in reference [28] is a special kind of materials. The strain-stiffening behavior happens when the tissue is stretched in the fiber directions, while it becomes strain-softening when stretched in transverse directions. One value of the exponent " $m$ " cannot predict this phenomenon.
4.2. Simple Shear. We consider a homogeneous simple shear in the isotropic plane of a transversely isotropic material. The deformation is defined by

$$
\begin{aligned}
& x_{1}=X_{1}, \\
& x_{2}=X_{2}+\gamma X_{3}, \\
& x_{3}=X_{3},
\end{aligned}
$$

where $\left(X_{1}, X_{2}, X_{3}\right)$ and $\left(x_{1}, x_{2}, x_{3}\right)$ are the coordinates of a point before and after deformation, respectively. The principal stretches are

$$
\begin{aligned}
\Lambda_{1} & =1 \\
\Lambda_{2,3} & =\frac{1}{2}\left(2+\gamma^{2} \mp \gamma \sqrt{4+\gamma^{2}}\right) .
\end{aligned}
$$

Let $\mathbf{l}_{0}=\mathbf{e}_{1}$. Then, from (28), (29), and (13), the physical components of Cauchy stress tensor can be expressed as

$$
\begin{aligned}
& \sigma_{11}=(a+b)\left(f_{2}+f_{3}\right) \\
& \sigma_{22}=\frac{m(a+2 b)\left[f_{2}^{2}\left(1+\Lambda_{2}\right)+f_{3}^{2}\left(1+\Lambda_{3}\right)\right]+2 b\left[f_{2}\left(1+\Lambda_{2}\right)+f_{3}\left(1+\Lambda_{3}\right)\right]}{4+\gamma^{2}}+a\left(f_{2}+f_{3}+m f_{2} f_{3}\right) \\
& \sigma_{33}=\frac{m(a+2 b)\left[f_{3}^{2}\left(1+\Lambda_{2}\right)+f_{2}^{2}\left(1+\Lambda_{3}\right)\right]+2 b\left[f_{3}\left(1+\Lambda_{2}\right)+f_{2}\left(1+\Lambda_{3}\right)\right]}{4+\gamma^{2}}+a\left(f_{2}+f_{3}+m f_{2} f_{3}\right) \\
& \sigma_{23}=-\frac{2 b\left(f_{2}-f_{3}\right)+m(a+2 b)\left(f_{2}^{2}-f_{3}^{2}\right)}{\sqrt{4+\gamma^{2}}} .
\end{aligned}
$$

Next, we will study how the stress $\sigma_{33}$ in (61) will change in the case of infinitesimal deformations. Consider the Taylor expansion of the third equation of (61) for a very small $\gamma$, and then

$$
\sigma_{33}=\frac{1}{4}[m(a+3 b)-2 b] \gamma^{2}+o\left(\gamma^{4}\right)
$$

It may be clear that, for a small shear deformation, each Hill's model (26) with

$$
m>\frac{2 b}{a+3 b}=\frac{2 \zeta}{2\left(v+\xi v^{\prime 2}\right)+3 \zeta}
$$

will predict the compressive stress, whereas each Hill's model (26) with

$$
m<\frac{2 \zeta}{2\left(v+\xi v^{\prime 2}\right)+3 \zeta}
$$

will predict the tensile stress. When $m=2 b /(a+3 b), \sigma_{33}$ will vanish for small deformation cases.

Figure 5 plots the developments of the nondimensional stress $\sigma_{33} / b$ in the isotropic plane shear deformation for the transversely isotropic Hill model with $m=0, \pm 1, \pm 2, \pm 3$. Figure 5 adopts the same material parameters as those of
Figure 1, such that $2 \zeta /\left(2\left(v+\xi v^{\prime 2}\right)+3 \zeta\right)=0.29$. It can be seen that when $m<0.29$ the material is subjected to pressure in the $x_{3}$ direction, and when $m>0.29$ the material is subjected to tension. These results are consistent with those of (63) and (64) in the infinitesimal deformation.

Next we will show how $m$ affects the shear stress $\sigma_{23} / b$ in the isotropic plane shear deformation. Taking the same parameters as those of Figure 1, we present in Figure 6 the developments of the nondimensional stress $\sigma_{23} / b$ for $m=$ $0, \pm 1, \pm 2, \pm 3$. It is shown that $\sigma_{23} / b$ takes the same values for $m$ and $-m$. When $m>0$ the stress $\sigma_{23} / b$ stiffens in the shear process; for $m=0$ it will reach its upper bound at $\gamma=3.29$.

\section{Conclusion}

Based on the structure tensor method, the present paper generalized the isotropic Hill theory to transversely isotropic media undergoing moderate deformations. The differences of these models are discussed through the analyses of the uniaxial stretch and the simple shear problems. Such generalizations of the structures of the isotropic theory to the transversely isotropic cases might provide an efficient method to model the stiffening or softening behaviors of materials 


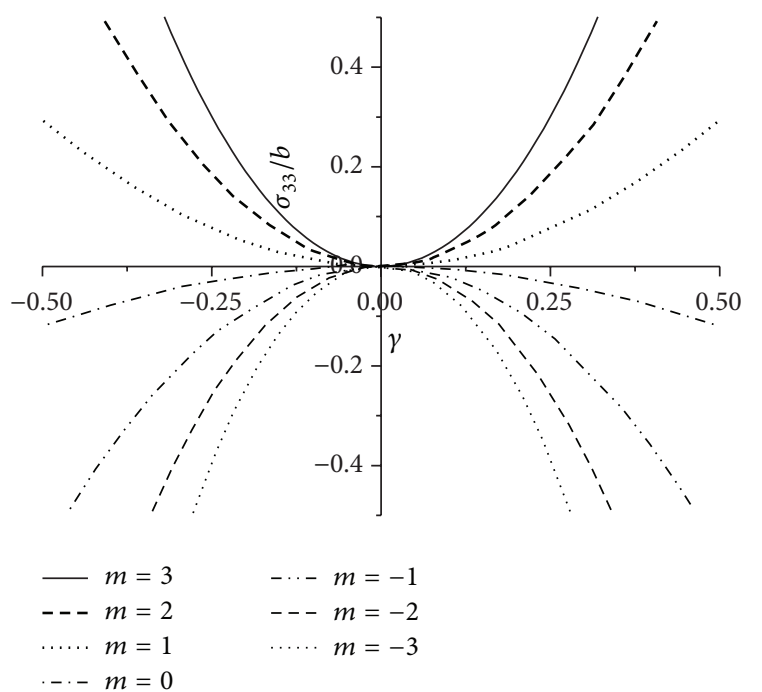

FIGURE 5: Relations between nondimensional normal stress $\sigma_{33} / b$ and shear deformation $\gamma$ (shear in the isotropic plane).

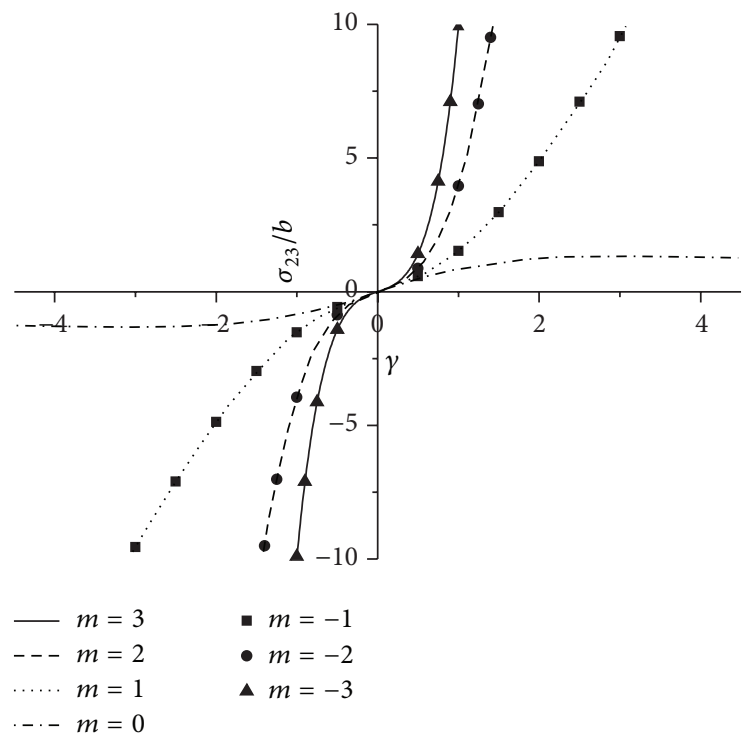

FIGURE 6: Relations between nondimensional shear stress $\sigma_{23} / b$ and shear deformation $\gamma$ (shear in the isotropic plane).

at moderate deformations. The results may be useful for the mechanical analysis of soft tissue materials.

\section{Competing Interests}

The authors declare that there is no conflict of interests regarding the publication of this paper.

\section{Acknowledgments}

This work is supported by National Natural Science Foundation of China (nos. 11472249 and 41572360), the Fundamental Research Funds for the Central Universities (no. 292015080), the Beijing Higher Education Young Elite Teacher Project (nos. YETP0645 and YETP0648), and the National Key Technology Support Program of China (no. 2015BAD20B02). Meanwhile, great thanks also go to former researchers for their excellent works, which give great help for the authors' academic study.

\section{References}

[1] R. C. Batra, "Linear constitutive relations in isotropic finite elasticity," Journal of Elasticity, vol. 51, no. 3, pp. 243-245, 1998.

[2] R. C. Batra, "Comparison of results from four linear constitutive relations in isotropic finite elasticity," International Journal of Non-Linear Mechanics, vol. 36, no. 3, pp. 421-432, 2001.

[3] A. Chiskis and R. Parnes, "Linear stress-strain relations in nonlinear elasticity," Acta Mechanica, vol. 146, pp. 109-113, 2000.

[4] R. Hill, "Aspects of invariance in solid mechanics," Advances in Applied Mechanics, vol. 18, pp. 1-75, 1978.

[5] J. J. Nader, "Linear response in finite elasticity" Journal of Elasticity, vol. 73, no. 1-3, pp. 165-172, 2003.

[6] C. Miehe and M. Lambrecht, "Algorithms for computation of stresses and elasticity moduli in terms of Seth-Hill's family of generalized strain tensors," Communications in Numerical Methods in Engineering, vol. 17, no. 5, pp. 337-353, 2001.

[7] H. Xiao and L. S. Chen, "Hencky's elasticity model and linear stress-strain relations in isotropic finite hyperelasticity," Acta Mechanica, vol. 157, no. 1-4, pp. 51-60, 2002.

[8] H. Xiao and L.-S. Chen, "Henky's logarithmic strain and dual stress-strain and strain-stress relations in isotropic finite hyperelasticity," International Journal of Solids and Structures, vol. 40, no. 6, pp. 1455-1463, 2003.

[9] K. Farahani and H. Bahai, "Hyper-elastic constitutive equations of conjugate stresses and strain tensors for the Seth-Hill strain measures," International Journal of Engineering Science, vol. 42, no. 1, pp. 29-41, 2004.

[10] H. Xiao and L. H. He, "A unified exact analysis for the Poynting effects of cylindrical tubes made of Hill's class of Hookean compressible elastic materials at finite strain," International Journal of Solids and Structures, vol. 44, no. 2, pp. 718-731, 2007.

[11] J. G. Murphy, "Linear isotropic relations in finite hyperelasticity: some general results," Journal of Elasticity, vol. 86, no. 2, pp. 139154, 2007.

[12] M. D. Gilchrist, J. G. Murphy, and B. Rashid, "Generalisations of the strain-energy function of linear elasticity to model biological soft tissue," International Journal of Non-Linear Mechanics, vol. 47, no. 2, pp. 268-272, 2012.

[13] J. L. Ericksen and R. S. Rivlin, "Large elastic deformations of homogeneous anisotropic materials," Archive for Rational Mechanics and Analysis, vol. 3, pp. 281-301, 1954.

[14] A. J. M. Spencer, Deformations of Fiber-Reinforced Materials, Oxford University Press, London, UK, 1972.

[15] M. Silhavy, The Mechanics and Thermodynamics of Continuous Media, Springer, Berlin, Germany, 1997.

[16] G. A. Holzapfel, Nonlinear Solid Mechanics: A Continuum Approach for Engineering, John Wiley \& Sons, 2000.

[17] J. Bonet and A. J. Burton, "A simple orthotropic, transversely isotropic hyperelastic constitutive equation for large strain computations," Computer Methods in Applied Mechanics and Engineering, vol. 162, no. 1-4, pp. 151-164, 1998. 
[18] R. W. Ogden, "Elements of the theory of finite elasticity," in Nonlinear Elasticity: Theory and Applications, Y. B. Fu and R. W. Ogden, Eds., vol. 283 of London Mathematical Society Lecture Note Series, pp. 1-57, Cambridge University Press, Cambridge, UK, 2001.

[19] Q. S. Zheng, "Theory of representations for tensor functionsa unified invariant approach to constitutive equations," Applied Mechanics Reviews, vol. 47, no. 11, pp. 545-587, 1994.

[20] G. Chagnon, M. Rebouah, and D. Favier, "Hyperelastic energy densities for soft biological tissues: a review," Journal of Elasticity, vol. 120, no. 2, pp. 129-160, 2015.

[21] J. Merodio and G. Saccomandi, "Remarks on cavity formation in fiber-reinforced incompressible non-linearly elastic solids," European Journal of Mechanics A: Solids, vol. 25, no. 5, pp. 778792, 2006.

[22] J. Merodio, R. W. Ogden, and J. Rodríguez, "The influence of residual stress on finite deformation elastic response," International Journal of Non-Linear Mechanics, vol. 56, pp. 43-49, 2013.

[23] A. Menzel and P. Steinmann, "On the comparison of two strategies to formulate orthotropic hyperelasticity," Journal of Elasticity, vol. 62, no. 3, pp. 171-201, 2001.

[24] R. Mahnken, "Anisotropy in geometrically non-linear elasticity with generalized Seth-Hill strain tensors projected to invariant subspaces," Communications in Numerical Methods in Engineering, vol. 21, no. 8, pp. 405-418, 2005.

[25] P. Papadopoulos and J. Lu, "On the formulation and numerical solution of problems in anisotropic finite plasticity," Computer Methods in Applied Mechanics and Engineering, vol. 190, no. 3738, pp. 4889-4910, 2001.

[26] C. Miehe, N. Apel, and M. Lambrecht, "Anisotropic additive plasticity in the logarithmic strain space: modular kinematic formulation and implementation based on incremental minimization principles for standard materials," Computer Methods in Applied Mechanics and Engineering, vol. 191, no. 47-48, pp. 5383-5425, 2002.

[27] A. Menzel, "Relations between material, intermediate and spatial generalized strain measures for anisotropic multiplicative inelasticity," Acta Mechanica, vol. 182, no. 3-4, pp. 231-252, 2006.

[28] M. Takaza, K. M. Moerman, J. Gindre, G. Lyons, and C. K. Simms, "The anisotropic mechanical behaviour of passive skeletal muscle tissue subjected to large tensile strain," Journal of the Mechanical Behavior of Biomedical Materials, vol. 17, pp. 209220, 2012.

[29] R. W. Ogden, Non-Linear Elastic Deformations, Ellis Horwood, Chichester, UK, 1984. 


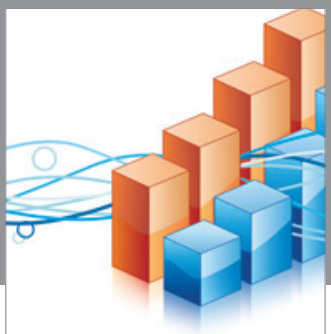

Advances in

Operations Research

vatem alat4

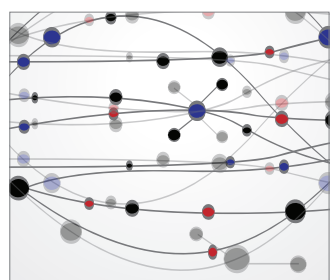

\section{The Scientific} World Journal
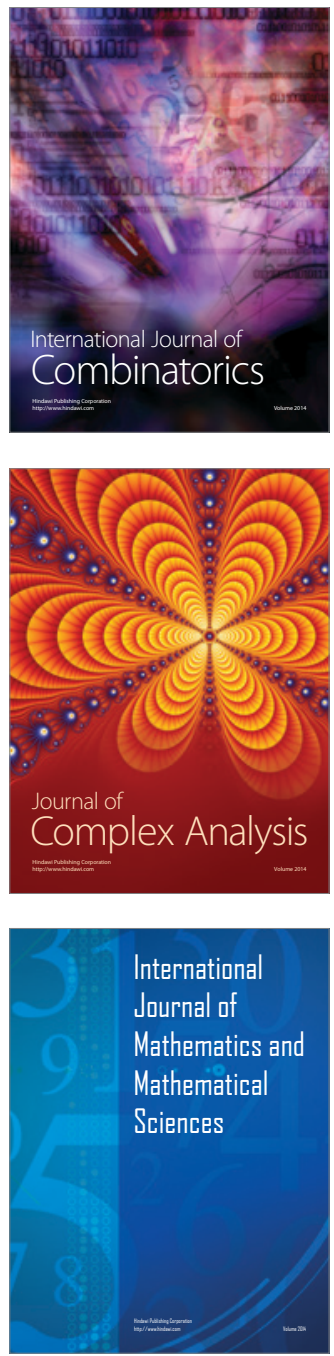
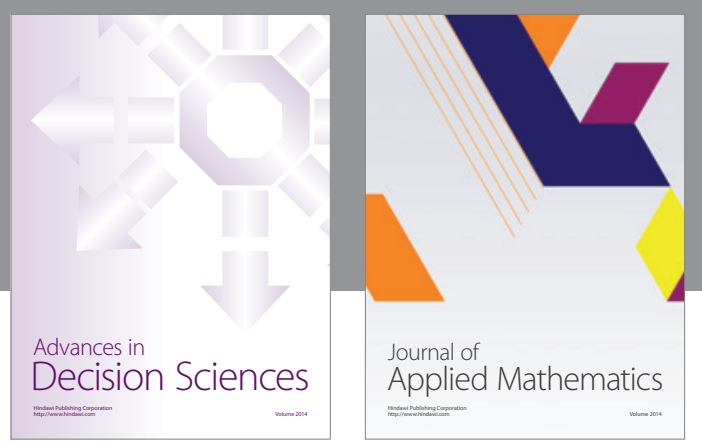

Algebra

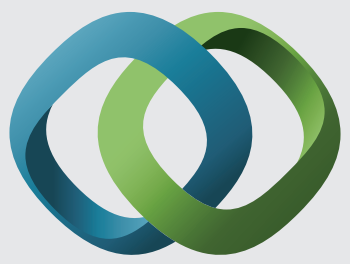

\section{Hindawi}

Submit your manuscripts at

http://www.hindawi.com
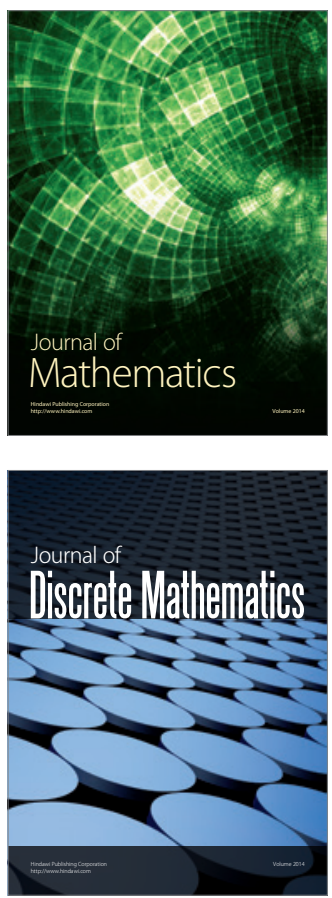

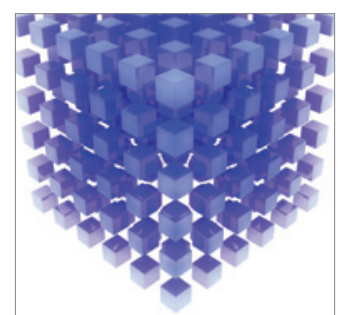

Mathematical Problems in Engineering
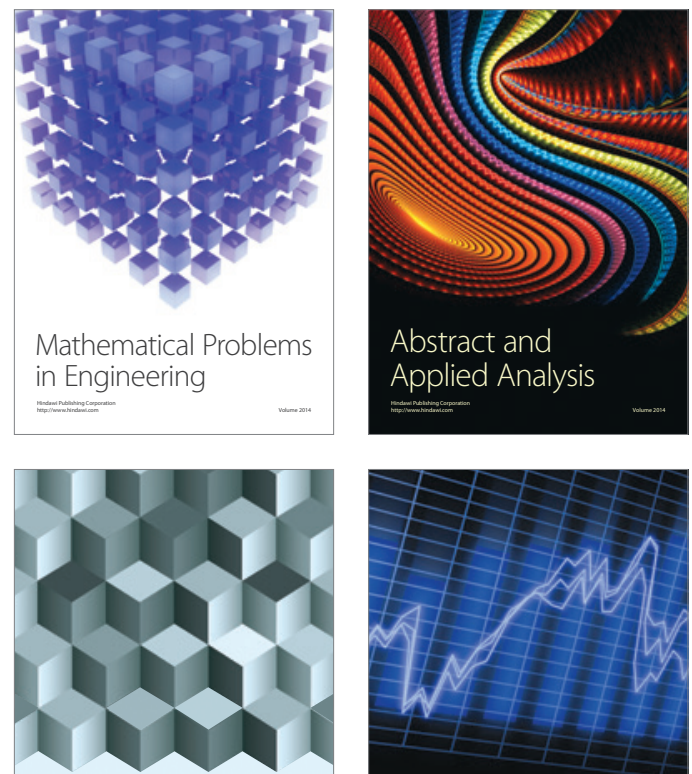

Journal of

Function Spaces

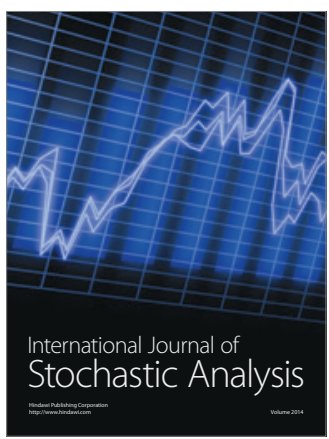

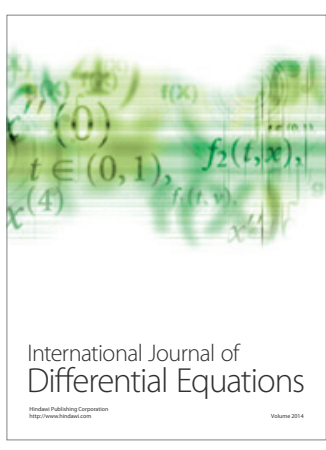
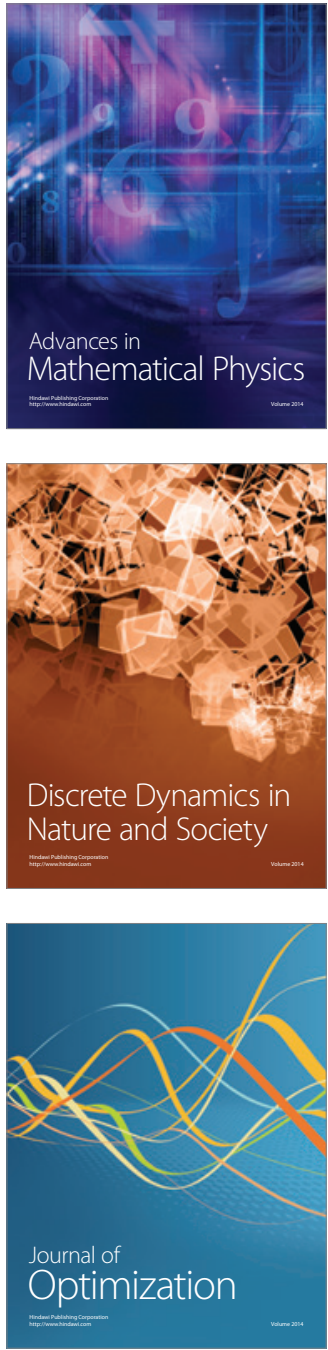